\title{
New generalized Pólya-Szegö and Čebyšev type inequalities with general kernel and measure
}

\author{
S. Iqbal ${ }^{1}$, M. Samraiz² Thabet Abdeljawad $^{3,4,5^{*}}$ (D), Kottakkaran Sooppy Nisar ${ }^{6}$, G. Rahman ${ }^{7}$ and M. Adil \\ Khan $^{8}$
}

"Correspondence:

tabdeljawad@psu.edu.sa

${ }^{3}$ Department of Mathematics and

General Sciences, Prince Sultan

University, Riyadh 12345, Saudi

Arabia

${ }^{4}$ Department of Medical Research,

China Medical University, Taichung

40402, Taiwan

Full list of author information is

available at the end of the article

\section{Springer}

\begin{abstract}
It is always attractive and motivating to acquire the generalizations of known results. In this article, we introduce a new class $\mathfrak{C}(\mathfrak{h})$ of functions which can be represented in a form of integral transforms involving general kernel with $\sigma$-finite measure. We obtain some new Pólya-Szegö and Čebyšev type inequalities as generalizations to the previously proved ones for different fractional integrals including fractional integral of a function with respect to another function capturing Riemann-Liouville integrals, Hadamard fractional integrals, Katugampola fractional integral operators, and conformable fractional integrals. This new idea shall motivate the researchers to prove the results over a measure space with general kernels instead of special kernels.
\end{abstract}

MSC: 26D15; 26D10; 26A33; 34B27

Keywords: Pólya-Szegö type inequalities; Čebyšev type inequalities; General kernel; $\sigma$-finite measure; Fractional integrals

\section{Introduction}

There are several problems in the mathematics and its related real world applications wherein fractional derivatives occupy an important place [1-13]. In many technologies, the fractional derivatives take a place in a way they can be described in different approaches, where these approaches can be used to explain a lot of essential real world problems. Each conventional fractional operator with its own special kernel can be used in a certain problem. Analyzing the uniqueness of fractional ordinary and partial differential equations can be performed by employing fractional integral inequalities. In the literature many applications can be found (for example, see [14-20]). For broader applications, we recommend the reader to see [21-29]. Recently, many authors have utilized unique versions of such inequalities to study diverse classes of differential and integral equations. Such types of inequalities are considered as far-reaching tools that demonstrate the analytical properties of several classes of differential and integral equations [30-41]. Čebyšev [42] came up with his well-known celebrated functional as follows:

(c) The Author(s) 2020. This article is licensed under a Creative Commons Attribution 4.0 International License, which permits use, sharing, adaptation, distribution and reproduction in any medium or format, as long as you give appropriate credit to the original author(s) and the source, provide a link to the Creative Commons licence, and indicate if changes were made. The images or other third party material in this article are included in the article's Creative Commons licence, unless indicated otherwise in a credit line to the material. If material is not included in the article's Creative Commons licence and your intended use is not permitted by statutory regulation or exceeds the permitted use, you will need to obtain permission directly from the copyright holder. To view a copy of this licence, visit http://creativecommons.org/licenses/by/4.0/. 
For Lebesgue integrable functions $\chi_{1}, \chi_{2}:\left[a_{1}, a_{2}\right] \rightarrow \mathbb{R}$, we consider the Čebyšev functional

$$
\begin{aligned}
\Omega\left(\chi_{1}, \chi_{2}\right)= & \frac{1}{a_{2}-a_{1}} \int_{a_{1}}^{a_{2}} \chi_{1}(\rho) \chi_{2}(\rho) d \rho \\
& -\frac{1}{a_{2}-a_{1}} \int_{a_{1}}^{a_{2}} \chi_{1}(\rho) d \rho \cdot \frac{1}{a_{2}-a_{1}} \int_{a_{1}}^{a_{2}} \chi_{2}(\rho) d \rho,
\end{aligned}
$$

where $\chi_{1}$ and $\chi_{2}$ are two integrable functions on $\left[a_{1}, a_{2}\right]$. If $\chi_{1}$ and $\chi_{2}$ are synchronous, that is,

$$
\left[\chi_{1}(\zeta)-\chi_{1}(\varsigma)\right]\left[\chi_{2}(\zeta)-\chi_{2}(\varsigma)\right] \geq 0
$$

for any $\gamma, \omega \in\left[a_{1}, a_{2}\right]$, then $\Omega\left(\chi_{1}, \chi_{2}\right) \geq 0$.

An enormous amount of efforts have been devoted to sharpening and extension of the classical inequalities. The inequality presented in the following definition is one of the interesting parts of the theory of classical inequalities known as Grüss type inequality [35]

$$
\left|\Omega\left(\chi_{1}, \chi_{2}\right)\right| \leq \frac{(C-c)(D-d)}{4},
$$

where $\chi_{1}$ and $\chi_{2}$ are integrable functions that satisfy

$$
c \leq \chi_{1}(\zeta) \leq C
$$

and

$$
d \leq \chi_{2}(\zeta) \leq D
$$

for all $\gamma \in\left[a_{1}, a_{2}\right]$ and for some $c, C, d, D \in \mathbb{R}$.

Pólya-Szegö introduced one of the most intensively studied inequalities in [43] stated as follows:

$$
\frac{\int_{a_{1}}^{a_{2}} \chi_{1}^{2}(\zeta) d \zeta \int_{a_{1}}^{a_{2}} \chi_{2}^{2}(\zeta) d \zeta}{\int_{a_{1}}^{a_{2}} \chi_{1}(\zeta) \chi_{2}(\zeta) d \zeta} \leq \frac{1}{4}\left(\sqrt{\frac{c d}{C D}}+\sqrt{\frac{C D}{c d}}\right)^{2} .
$$

The constant $\frac{1}{4}$ is least possible such that inequality (1.2) is valid.

Dragomir and Diamond in [44] proved the following Grüss type inequality via inequality (1.2) by utilizing the Pólya-Szegö inequality:

$$
\left|\Omega\left(\chi_{1}, \chi_{2}\right)\right| \leq \frac{(C-c)(D-d)}{4\left(a_{2}-a_{1}\right) \sqrt{c d C D} \int_{a_{1}}^{a_{2}} \chi_{1}^{2}(\zeta) d \zeta \int_{a_{1}}^{a_{2}} \chi_{2}^{2}(\zeta) d \zeta} .
$$

Let $(\Delta, \Sigma, \beta)$ be measure space with positive $\sigma$-finite measure, $h: \Delta \times \Delta \rightarrow \mathbb{R}$ be a nonnegative function, and

$$
\Upsilon(\varsigma)=\int_{\Delta} h(\varsigma, \zeta) d \beta(\zeta), \quad \varsigma \in \Delta .
$$

Throughout this paper, we suppose $\Upsilon(\varsigma)>0$ a.e. on $\Delta$. 
Let $\mathfrak{C}(\mathfrak{h})$ denote the class of functions $\mho: \Delta \rightarrow \mathbb{R}$ with the representation

$$
\bar{\mho}(\varsigma)=\int_{\Delta} h(\varsigma, \zeta) \mho(\zeta) d \beta(\zeta)
$$

where $\bar{\mho}: \Delta \rightarrow \mathbb{R}$ is a measurable function.

Definition 1.1 (see $[13,45]$ ) Let $\vartheta \in L_{1}[a, b]$ and $\mathfrak{R}_{a^{+}}^{\varrho} \vartheta$ and $\mathfrak{R}_{b^{-}}^{\varrho} \vartheta$ be the left-sided and right-sided Riemann-Liouville fractional integrals of order $\varrho>0$ defined by

$$
\mathfrak{R}_{a^{+}}^{\varrho} \vartheta(\varsigma)=\frac{1}{\Gamma(\varrho)} \int_{a}^{\varsigma} \vartheta(\zeta)(\varsigma-\zeta)^{\varrho-1} d \zeta \quad(\varsigma>a)
$$

and

$$
\mathfrak{R}_{b^{-}}^{\varrho} \vartheta(\varsigma)=\frac{1}{\Gamma(\varrho)} \int_{\varsigma}^{b} \vartheta(\zeta)(\zeta-\varsigma)^{\varrho-1} d \zeta \quad(\varsigma<b),
$$

respectively, where $\Gamma(\varrho)=\int_{0}^{\infty} e^{-\wp}-\wp^{\varrho-1} d \wp$ is the usual gamma function.

Diaz et al. in [46] originated the following definition of gamma $k$-function.

Definition 1.2 The generalized $\Gamma$ function known as $\Gamma-k$ function is defined by the following definition:

$$
\Gamma_{k}(\zeta)=\lim _{m \rightarrow \infty} \frac{m ! k^{m}(m k)^{\frac{t}{k}-1}}{(\zeta)_{m, k}}, \quad k>0, \mathbb{R}(\zeta)>0
$$

where $(\zeta)_{m, k}=t(\zeta+k)(\zeta+2 k) \ldots,(\zeta+(m-1) k), m \geq 1$, is Pochhammer $k$ symbol. The generalized gamma function can also be written as

$$
\Gamma_{k}(\zeta)=\int_{0}^{\infty} x^{\zeta-1} e^{\frac{-x^{k}}{k}} d x, \quad \mathbb{R}(\zeta)>0
$$

Specially, for $k=1, \Gamma_{1}(\zeta)=\Gamma(\zeta)$.

Definition 1.3 ([47]) Let $f \in L_{1}([a, b])$ (the Lebesgue measure). The left-sided and rightsided Riemann-Liouville $k$-fractional integrals $\mathfrak{R}_{a^{+}}^{\varrho, k} f$ and $\mathfrak{R}_{b^{-}}^{\varrho, k} f$ of order $\varrho>0$ are defined by

$$
\mathfrak{R}_{a^{+}}^{\varrho, k} f(\varsigma)=\frac{1}{k \Gamma_{k}(\varrho)} \int_{a}^{\varsigma} f(\zeta)(\varsigma-\zeta)^{\frac{\varrho}{k}-1} d \zeta \quad(\varsigma>a)
$$

and

$$
\mathfrak{R}_{b^{-}}^{\varrho, k} f(\varsigma)=\frac{1}{k \Gamma_{k}(\varrho)} \int_{\varsigma}^{b} f(\zeta)(\zeta-\varsigma)^{\frac{\varrho}{k}-1} d \zeta \quad(\varsigma<b),
$$

where $\Gamma_{k}(\cdot)$ is the $k$-gamma function. 
In 2006 Kilbas et al. in [48] presented the definition of fractional integrals concerning another function as follows.

Definition 1.4 Let $(a, b),-\infty \leq a<b \leq \infty$, and $\varrho>0 \psi$ be a positive increasing function on $(a, b]$. The left-sided and right-sided fractional integrals of a function $f$ concerning another function $\psi$ in $[a, b]$ are given by

$$
I_{a+; \psi}^{\varrho} f(\varsigma)=\frac{1}{\Gamma(\varrho)} \int_{a}^{\varsigma} \frac{\psi^{\prime}(\zeta) f(\zeta) d \zeta}{[\psi(\varsigma)-\psi(\zeta)]^{1-\varrho}}, \quad \varsigma>a,
$$

and

$$
I_{b-; \psi}^{\varrho} f(\varsigma)=\frac{1}{\Gamma(\varrho)} \int_{x}^{b} \frac{\psi^{\prime}(\zeta) f(\zeta) d \zeta}{[\psi(\zeta)-\psi(\varsigma)]^{1-\varrho}}, \quad \varsigma<b
$$

The classical Hadamard fractional integral and its generalized form is given in the next two definitions.

Definition 1.5 Let $(a, b)$ be a finite or infinite interval of the half axis $\mathbb{R}_{+}$and $\varrho>0$. The Hadamard-type fractional integrals of order $\varrho>0$ are given by

$$
J_{a_{+}}^{\varrho} f(\varsigma)=\frac{1}{\Gamma(\varrho)} \int_{a}^{\varsigma}\left(\log \frac{\varsigma}{\zeta}\right)^{\varrho-1} \frac{f(\zeta) d \zeta}{\zeta}, \quad \varsigma>a,
$$

and

$$
J_{b-}^{\varrho} f(\varsigma)=\frac{1}{\Gamma(\varrho)} \int_{x}^{b}\left(\log \frac{\zeta}{\zeta}\right)^{\varrho-1} \frac{f(\zeta) d \zeta}{\zeta}, \quad \varsigma<b
$$

respectively.

The generalized Hadamard type fractional integrals are stated in the next definition.

Definition 1.6 Let $(a, b)$ be a finite or infinite interval of the half axis $\mathbb{R}_{+}$and $\varrho>0$. The Hadamard fractional integrals of order $\varrho>0$ are given by

$$
J_{a_{+}}^{\varrho, k} f(\varsigma)=\frac{1}{\Gamma_{k}(\varrho)} \int_{a}^{\varsigma}\left(\log \frac{\varsigma}{\zeta}\right)^{\frac{\varrho}{k}-1} \frac{f(\zeta) d \zeta}{y}, \quad \varsigma>a,
$$

and

$$
J_{b_{-}}^{\varrho, k} f(\varsigma)=\frac{1}{\Gamma_{k}(\varrho)} \int_{x}^{b}\left(\log \frac{y}{x}\right)^{\frac{\varrho}{k}-1} \frac{f(\zeta) d \zeta}{y}, \quad \varsigma<b
$$

respectively.

Recently, Saima et al. in [49] generalized the definition of operator given in Definition 1.4 stated as follows. 
Definition 1.7 Let $(a, b)$ be a finite or infinite interval on the real line $\Re$ together with $k>0$. Let $\psi>0$ be an increasing function on $(a, b]$, then the generalized fractional integrals of a function $f$ concerning another function $\psi$ of order $\varrho>0$ are given by

$$
I_{a+; \psi}^{\varrho, k} f(\varsigma)=\frac{1}{\Gamma_{k}(\varrho)} \int_{a}^{\varsigma} \frac{\psi^{\prime}(\zeta) f(\zeta) d \rho}{[\psi(\varsigma)-\psi(\zeta)]^{1-\frac{\varrho}{k}}}, \quad \varsigma>a,
$$

and

$$
I_{b-; \psi}^{\varrho, k} f(\varsigma)=\frac{1}{\Gamma(\varrho)} \int_{x}^{b} \frac{\psi^{\prime}(\zeta) f(\zeta) d \rho}{[\psi(\zeta)-\psi(\varsigma)]^{1-\frac{\varrho}{k}}}, \quad \varsigma<b
$$

Note that corresponding to $\psi(\varsigma)=\log (\varsigma)$ the above defined operator represents the Hadamard fractional integrals of order $\varrho$.

The definition of the Erdélyi-Köber fractional integrals is given by the following definition. For details, we refer the reader to the book [50].

Definition 1.8 Let $(a, b)$ be a finite or infinite interval of the half axis $\mathbb{R}_{+}$together with $\varrho, \sigma>0$ and $\eta \in \mathbb{R}$. The left-sided and right-sided integrals of order $\varrho \in \mathbb{R}$ are defined by

$$
I_{a_{+} ; \sigma ; \eta}^{\varrho} f(\varsigma)=\frac{\sigma \varsigma^{-\sigma(\varrho+\eta)}}{\Gamma(\varrho)} \int_{a}^{\varsigma} \frac{\zeta^{\sigma \eta+\sigma-1} f(\zeta) d \zeta}{\left(\varsigma^{\sigma}-\zeta^{\sigma}\right)^{1-\varrho}}
$$

and

$$
I_{b_{-} ; \sigma ; \eta}^{\varrho} f(\varsigma)=\frac{\sigma \zeta^{\sigma \eta}}{\Gamma(\varrho)} \int_{\varsigma}^{b} \frac{z e t a^{\sigma(1-\eta-\varrho)-1} f(\zeta) d \zeta}{\left(\zeta^{\sigma}-\zeta^{\sigma}\right)^{1-\varrho}}
$$

respectively.

Consider the space $X_{c}^{p}(a, b),(c \in \mathbb{R}, 1 \leq p \leq \infty)$ of complex-valued Lebesgue measurable functions $f$ on $[a, b]$ such that $\|f\|_{X_{c}^{p}(a, b)}<\infty$, where

$$
\|f\|_{X_{c}^{p}}=\left(\int_{a}^{b}\left|\varsigma^{c} f(\varsigma)\right|^{p} \frac{d \varsigma}{\varsigma}\right)<\infty
$$

Definition 1.9 Let $[a, b] \subset \mathbb{R}$ be a finite interval. Then the left- and right-sided Katugampola fractional integrals of order $\varrho>0$ of $f \in X_{c}^{p}(a, b)$ are defined by

$$
{ }^{\rho} I_{a_{+}}^{\varrho} f(\varsigma)=\frac{\rho^{1-\varrho}}{\Gamma(\varrho)} \int_{a}^{\varsigma} \frac{\zeta^{\rho-1} f(\zeta) d \zeta}{\left(\varsigma^{\rho}-\zeta^{\rho}\right)^{1-\varrho}}
$$

and

$$
{ }^{\rho} I_{b}^{\varrho} f(\varsigma)=\frac{\rho^{1-\varrho}}{\Gamma(\varrho)} \int_{\varsigma}^{b} \frac{\zeta^{\rho-1} f(\zeta) d \zeta}{\left(\zeta^{\rho}-\varsigma^{\rho}\right)^{1-\varrho}}
$$

with $a<n u<b$ and $\rho>0$, if the integral exists. 
Definition 1.10 ([51]) Let $\beta \in \mathbb{C}, \mathbb{R}(\beta)>0$. We define the left-fractional conformable integral operator by

$$
{ }_{\beta}^{\rho} \mathfrak{J}^{\varrho} f(\varsigma)=\frac{1}{\Gamma(\beta)} \int_{a}^{\zeta}\left(\frac{(\zeta-a)^{\varrho}-(\zeta-a)^{\varrho}}{\varrho}\right)^{\beta-1} f(\zeta) \frac{d \zeta}{(\zeta-a)^{1-\varrho}}
$$

and

$$
{ }_{\beta}^{\rho} \mathfrak{J}^{\varrho} f(\varsigma)=\frac{1}{\Gamma(\beta)} \int_{\varsigma}^{b}\left(\frac{(b-\varsigma)^{\varrho}-(b-\zeta)^{\varrho}}{\varrho}\right)^{\beta-1} f(\zeta) \frac{d \zeta}{(b-\zeta)^{1-\varrho}} .
$$

Definition 1.11 ([52]) Let $\phi$ be a confirmable fractional integral on the interval $[p, q] \subseteq$ $(0, \infty)$. The right-sided and left-sided generalized conformable fractional integrals ${ }_{\varrho}^{\tau} K_{p^{+}}^{\beta}$ and ${ }_{\varrho}^{\tau} K_{q^{-}}^{\beta}$ of order $\beta>0, \tau \in \mathbb{R}, \varrho+\tau \neq 0$, are defined by

$$
{ }_{\varrho}^{\tau} K_{p^{+}}^{\beta} \phi(\zeta)=\frac{1}{\Gamma(\beta)} \int_{p}^{\zeta}\left(\frac{\zeta^{\varrho+\tau}-\varsigma^{\varrho+\tau}}{\varrho+\tau}\right)^{\beta-1} \phi(\varsigma) \varsigma^{\tau} d_{\varrho} \varsigma
$$

and

$$
{ }_{\varrho}^{\tau} K_{q_{-}}^{\beta} \phi(\zeta)=\frac{1}{\Gamma(\beta)} \int_{\zeta}^{q}\left(\frac{\varsigma^{\varrho+\tau}-\zeta^{\varrho+\tau}}{\varrho+\tau}\right)^{\beta-1} \phi(\varsigma) \varsigma^{\tau} d_{\varrho} \varsigma
$$

respectively, ${ }_{\varrho}^{\tau} K_{p^{+}}^{0} \phi(\zeta)={ }_{\varrho}^{\tau} K_{q_{-}}^{0} \phi(\zeta)=\phi(\zeta)$.

\section{Main results}

In the present section, we set up the Pólya-Szegö and Cebyšev type inequalities for the general kernel with related applications in fractional calculus.

Theorem 2.1 Let $(\Delta, \Sigma, \beta)$ be a measure space with positive $\sigma$-finite measure. Let $h: \Delta \times$ $\Delta \rightarrow \mathbb{R}$ be nonnegative and $\chi_{1}, \chi_{2}, \Upsilon_{1}, \Upsilon_{2}, \Omega_{1}, \Omega_{2} \in \mathfrak{C}(\mathfrak{h})$ be positive integrable functions defined on $[0, \zeta)$ such that

$$
0<\Upsilon_{1}(\varsigma) \leq \chi_{1}(\varsigma) \leq \Upsilon_{2}(\varsigma), \quad 0<\Omega_{1}(\varsigma) \leq \chi_{2}(\varsigma) \leq \Omega_{2}(\varsigma)
$$

for all $\varsigma \in[0, \infty)$. Then

$$
\begin{aligned}
& \left(\overline{\left(\Upsilon_{1}(\zeta) \Omega_{1}(\zeta)+\Upsilon_{2}(\zeta) \Omega_{2}(\zeta)\right) \chi_{1}(\zeta) \chi_{2}(\zeta)}\right)^{2} \\
& \quad \geq 4 \overline{\Omega_{1}(\zeta) \Omega_{2}(\zeta) \chi_{1}^{2}(\zeta)} \cdot \overline{\Upsilon_{1}(\zeta) \Upsilon_{2}(\zeta) \chi_{2}^{2}(\zeta)} .
\end{aligned}
$$

Proof It follows from (2.1) that

$$
\left(\frac{\Upsilon_{2}(\varsigma)}{\Omega_{1}(\varsigma)}-\frac{\chi_{1}(\varsigma)}{\chi_{2}(\varsigma)}\right) \geq 0
$$

and

$$
\left(\frac{\chi_{1}(\varsigma)}{\chi_{2}(\varsigma)}-\frac{\Upsilon_{1}(\varsigma)}{\Omega_{2}(\varsigma)}\right) \geq 0 .
$$


Multiplying inequalities (2.3) and (2.4), we get

$$
\begin{aligned}
& {\left[\Upsilon_{1}(\varsigma) \Omega_{1}(\varsigma)+\Upsilon_{2}(\varsigma) \Omega_{2}(\varsigma)\right] \chi_{1}(\varsigma) \chi_{2}(\varsigma)} \\
& \quad \geq \Omega_{1}(\varsigma) \Omega_{2}(\varsigma) \chi_{1}^{2}(\varsigma)+\Upsilon_{1}(\varsigma) \Upsilon_{2}(\varsigma) \chi_{2}^{2}(\varsigma)
\end{aligned}
$$

Multiplying by $h(\zeta, \varsigma)$ and integrating with respect to $\varsigma$ over measure space $\Delta$, we get that

$$
\begin{aligned}
& \int_{\Delta} h(\zeta, \varsigma)\left[\Upsilon_{1}(\varsigma) \Omega_{1}(\varsigma)+\Upsilon_{2}(\varsigma) \Omega_{2}(\varsigma)\right] \chi_{1}(\varsigma) \chi_{2}(\varsigma) d \beta(\varsigma) \\
& \quad \geq \int_{\Delta} h(\zeta, \varsigma) \Omega_{1}(\varsigma) \Omega_{2}(\varsigma) \chi_{1}^{2}(\varsigma) d \beta(\varsigma)+\int_{\Delta} h(\zeta, \varsigma) \Upsilon_{1}(\varsigma) \Upsilon_{2}(\varsigma) \chi_{2}^{2}(\varsigma) d \beta(\varsigma) .
\end{aligned}
$$

Using the definition of $\mathfrak{C}(\mathfrak{h})$, we can write

$$
\begin{aligned}
& \overline{\left[\Upsilon_{1}(\zeta) \Omega_{1}(\zeta)+\Upsilon_{2}(\zeta) \Omega_{2}(\zeta)\right] \chi_{1}(\zeta) \chi_{2}(\zeta)} \\
& \quad \geq \overline{\Omega_{1}(\zeta) \Omega_{2}(\zeta) \chi_{1}^{2}(\zeta)}+\overline{\Upsilon_{1}(\zeta) \Upsilon_{2}(\zeta) \chi_{2}^{2}(\zeta)} .
\end{aligned}
$$

Now applying the arithmetic-geometric inequality, we have

$$
\begin{aligned}
& \overline{\left[\Upsilon_{1}(\zeta) \Omega_{1}(\zeta)+\Upsilon_{2}(\zeta) \Omega_{2}(\zeta)\right] \chi_{1}(\zeta) \chi_{2}(\zeta)} \\
& \quad \geq 2 \sqrt{\overline{\Omega_{1}(\zeta) \Omega_{2}(\zeta) \chi_{1}^{2}(\zeta)} \overline{\Upsilon_{1}(\zeta) \Upsilon_{2}(\zeta) \chi_{2}^{2}(\zeta)}},
\end{aligned}
$$

which leads to

$$
\begin{gathered}
\frac{1}{4}\left(\overline{\left[\Upsilon_{1}(\zeta) \Omega_{1}(\zeta)+\Upsilon_{2}(\zeta) \Omega_{2}(\zeta)\right] \chi_{1}(\zeta) \chi_{2}(\zeta)}\right)^{2} \\
\quad \geq \overline{\Omega_{1}(\zeta) \Omega_{2}(\zeta) \chi_{1}^{2}(\zeta)} \overline{\Upsilon_{1}(\zeta) \Upsilon_{2}(\zeta) \chi_{2}^{2}(\zeta)},
\end{gathered}
$$

which implies (2.2).

Corollary 2.2 Let $\zeta, c, d, C, D>0$ with $q \leq Q, r \leq R$, and let $\chi_{1}, \chi_{2}$ be two positive integrable functions defined on $[0, \infty)$ such that

$$
0<c \leq \chi_{1}(\varsigma) \leq C<\infty, \quad 0<d \leq \chi_{2}(\varsigma) \leq D<\infty
$$

for all $\varsigma \in[0, \infty)$. Then

$$
\frac{\overline{\chi_{1}^{2}(\zeta) \chi_{2}^{2}(\zeta)}}{\overline{\left(\chi_{1}(\zeta) \chi_{2}(\zeta)\right)^{2}}} \leq \frac{1}{4}\left(\sqrt{\frac{c d}{C D}}+\sqrt{\frac{C D}{c d}}\right)
$$

Corollary 2.3 Applying Theorem 2.1 with $\Delta=(a, b), d \beta(\varsigma)=d \varsigma$,

$$
h(\zeta, \varsigma)= \begin{cases}\frac{g^{\prime}(\varsigma)}{k \Gamma_{k}(\varrho)(g(\zeta)-g(\zeta))^{1-\frac{\rho}{k}}}, & a \leq \varsigma \leq \zeta \\ 0, & \zeta<\varsigma \leq b .\end{cases}
$$




\section{Replacing}

$$
\begin{aligned}
& \overline{\left(\Upsilon_{1}(\zeta) \Omega_{1}(\zeta)+\Upsilon_{2}(\zeta) \Omega_{2}(\zeta)\right) \chi_{1}(\zeta) \chi_{2}(\zeta)}=I_{a+; g}^{\varrho, k}\left(\Upsilon_{1}(\zeta) \Omega_{1}(\zeta)+\Upsilon_{2}(\zeta) \Omega_{2}(\zeta)\right) \chi_{1}(\zeta) \chi_{2}(\zeta), \\
& \overline{\Omega_{1}(\zeta) \Omega_{2}(\zeta) \chi_{1}^{2}(\zeta)}=I_{a+g}^{\rho, k} \Omega_{1}(\zeta) \Omega_{2}(\zeta) \chi_{1}^{2}(\zeta)
\end{aligned}
$$

and

$$
\overline{\Upsilon_{1}(\zeta) \Upsilon_{2}(\zeta) \chi_{2}^{2}(\zeta)}=I_{a+, g}^{\rho, k} \Upsilon_{1}(\zeta) \Upsilon_{2}(\zeta) \chi_{2}^{2}(\zeta),
$$

we get

$$
\begin{aligned}
& I_{a+; g}^{\rho, k}\left(\Upsilon_{1}(\zeta) \Omega_{1}(\zeta)+\Upsilon_{2}(\zeta) \Omega_{2}(\zeta)\right) \chi_{1}(\zeta) \chi_{2}(\zeta) \\
& \quad \geq 4 I_{a+; g}^{\rho, k} \Omega_{1}(\zeta) \Omega_{2}(\zeta) \chi_{1}^{2}(\zeta) \cdot I_{a+; g}^{\rho, k} \Upsilon_{1}(\zeta) \Upsilon_{2}(\zeta) \chi_{2}^{2}(\zeta)
\end{aligned}
$$

which is [49, Theorem 2.1].

Remark 2.4 In particular, if we choose $k=1$ in Corollary 2.3, we get the following inequality:

$$
\begin{aligned}
& I_{a+; g}^{\varrho}\left(\Upsilon_{1}(\zeta) \Omega_{1}(\zeta)+\Upsilon_{2}(\zeta) \Omega_{2}(\zeta)\right) \chi_{1}(\zeta) \chi_{2}(\zeta) \\
& \quad \geq 4 I_{a+; g}^{\varrho} \Omega_{1}(\zeta) \Omega_{2}(\zeta) \chi_{1}^{2}(\zeta) \cdot I_{a+; g}^{\varrho} \Upsilon_{1}(\zeta) \Upsilon_{2}(\zeta) \chi_{2}^{2}(\zeta),
\end{aligned}
$$

given in [49, Corollary 3.3].

Remark 2.5 Applying Corollary 2.3 with $\psi(\zeta)=\zeta$, and corresponding $h(\zeta, \varsigma)$ defined by (2.11) takes the form

$$
h(\zeta, \varsigma)= \begin{cases}\frac{1}{k \Gamma_{k}(\varrho)(\zeta-\varsigma)^{1-\frac{e}{k}},} & a \leq \varsigma \leq \zeta ; \\ 0, & \zeta<\varsigma \leq b,\end{cases}
$$

and (2.13) becomes

$$
\begin{aligned}
& I_{a+}^{\varrho, k}\left(\Upsilon_{1}(\zeta) \Omega_{1}(\zeta)+\Upsilon_{2}(\zeta) \Omega_{2}(\zeta)\right) \chi_{1}(\zeta) \chi_{2}(\zeta) \\
& \quad \geq 4 I_{a+}^{\varrho, k} \Omega_{1}(\zeta) \Omega_{2}(\zeta) \chi_{1}^{2}(\zeta) \cdot I_{a+}^{\varrho, k} \Upsilon_{1}(\zeta) \Upsilon_{2}(\zeta) \chi_{2}^{2}(\zeta),
\end{aligned}
$$

which leads to [49, Corollary 3.4]. Moreover, if we take $k=1$, then (2.15) becomes the inequality given in [36, Lemma 3.1].

Remark 2.6 Apply Theorem 2.1 with $\Delta=(a, b), d \beta(\varsigma)=d \varsigma$,

$$
h(\zeta, \varsigma)= \begin{cases}\frac{1}{{ } k \Gamma_{k}(\varrho)(\log \zeta-\log \varsigma)^{1-\frac{\varrho}{k}},} & a \leq \varsigma \leq \zeta ; \\ 0, & \zeta<\varsigma \leq b .\end{cases}
$$


Replacing

$$
\begin{aligned}
& \overline{\left(\Upsilon_{1}(\zeta) \Omega_{1}(\zeta)+\Upsilon_{2}(\zeta) \Omega_{2}(\zeta)\right) \chi_{1}(\zeta) \chi_{2}(\zeta)}=J_{a_{+}}^{\varrho}\left(\Upsilon_{1}(\zeta) \Omega_{1}(\zeta)+\Upsilon_{2}(\zeta) \Omega_{2}(\zeta)\right) \chi_{1}(\zeta) \chi_{2}(\zeta), \\
& \overline{\Omega_{1}(\zeta) \Omega_{2}(\zeta) \chi_{1}^{2}(\zeta)}=J_{a_{+}}^{\varrho} \Omega_{1}(\zeta) \Omega_{2}(\zeta) \chi_{1}^{2}(\zeta)
\end{aligned}
$$

and

$$
\overline{\Upsilon_{1}(\zeta) \Upsilon_{2}(\zeta) \chi_{2}^{2}(\zeta)}=J_{a_{+}}^{\varrho} \Upsilon_{1}(\zeta) \Upsilon_{2}(\zeta) \chi_{2}^{2}(\zeta)
$$

we get the following inequality involving Hadamard fractional integrals:

$$
\begin{aligned}
& J_{a+}^{\rho, k}\left(\Upsilon_{1}(\zeta) \Omega_{1}(\zeta)+\Upsilon_{2}(\zeta) \Omega_{2}(\zeta)\right) \chi_{1}(\zeta) \chi_{2}(\zeta) \\
& \quad \geq 4 J_{a+}^{\varrho, k} \Omega_{1}(\zeta) \Omega_{2}(\zeta) \chi_{1}^{2}(\zeta) \cdot J_{a+}^{\varrho, k} \Upsilon_{1}(\zeta) \Upsilon_{2}(\zeta) \chi_{2}^{2}(\zeta)
\end{aligned}
$$

Remark 2.7 Applying Theorem 2.1 with $\Delta=(a, b), d \beta(\varsigma)=d \varsigma$,

$$
h(\zeta, \varsigma)= \begin{cases}\frac{1}{\Gamma(\varrho)} \frac{\sigma \gamma^{-\sigma(\varrho+\eta)}}{\left(\gamma^{\sigma}-\varsigma^{\sigma}\right)^{1-\varrho}} \varsigma^{\sigma \eta+\sigma-1}, & a \leq \varsigma \leq \gamma \\ 0, & \gamma<\varsigma \leq b .\end{cases}
$$

If we use the following replacements:

$$
\begin{aligned}
& \overline{\left(\Upsilon_{1}(\zeta) \Omega_{1}(\zeta)+\Upsilon_{2}(\zeta) \Omega_{2}(\zeta)\right) \chi_{1}(\zeta) \chi_{2}(\zeta)} \\
& \quad=I_{a_{+} ; \sigma ; \eta}^{\varrho}\left(\Upsilon_{1}(\zeta) \Omega_{1}(\zeta)+\Upsilon_{2}(\zeta) \Omega_{2}(\zeta)\right) \chi_{1}(\zeta) \chi_{2}(\zeta), \\
& \overline{\Omega_{1}(\zeta) \Omega_{2}(\zeta) \chi_{1}^{2}(\zeta)}=I_{a_{+} ; \sigma ; \eta}^{\varrho} \Omega_{1}(\zeta) \Omega_{2}(\zeta) \chi_{1}^{2}(\zeta),
\end{aligned}
$$

and

$$
\overline{\Upsilon_{1}(\zeta) \Upsilon_{2}(\zeta) \chi_{2}^{2}(\zeta)}=I_{a_{+} ; \sigma ; \eta}^{\varrho} \Upsilon_{1}(\zeta) \Upsilon_{2}(\zeta) \chi_{2}^{2}(\zeta)
$$

then we get the following inequality involving Erdélyi-Köber fractional integral:

$$
\begin{aligned}
& I_{a_{+} ; \sigma ; \eta}^{\varrho}\left(\Upsilon_{1}(\zeta) \Omega_{1}(\zeta)+\Upsilon_{2}(\zeta) \Omega_{2}(\zeta)\right) \chi_{1}(\zeta) \chi_{2}(\zeta) \\
& \quad \geq 4 I_{a_{+} ; \sigma ; \eta}^{\varrho} \Omega_{1}(\zeta) \Omega_{2}(\zeta) \chi_{2}^{2}(\zeta) \cdot I_{a_{+} ; \sigma ; \eta}^{\varrho} \Upsilon_{1}(\zeta) \Upsilon_{2}(\zeta) \chi_{2}^{2}(\zeta) .
\end{aligned}
$$

Remark 2.8 Choosing $\beta>0, \psi(\zeta)=\frac{\gamma^{\beta}}{\beta}$, and $k=1$ in Corollary 2.3, we get the inequality for the Katugampola fractional integral operators in the literature [53] and the inequality takes the form

$$
\begin{aligned}
& { }^{\rho} I_{a_{+}}^{\varrho}\left(\Upsilon_{1}(\zeta) \Omega_{1}(\zeta)+\Upsilon_{2}(\zeta) \Omega_{2}(\zeta)\right) \chi_{1}(\zeta) \chi_{2}(\zeta) \\
& \quad \geq 4^{\rho} I_{a_{+}}^{\varrho} \Omega_{1}(\zeta) \Omega_{2}(\zeta) \chi_{1}^{2}(\zeta) \cdot{ }^{\rho} I_{a_{+}}^{\varrho} \Upsilon_{1}(\zeta) \Upsilon_{2}(\zeta) \chi_{2}^{2}(\zeta) .
\end{aligned}
$$

Remark 2.9 Choosing $\beta>0, \psi(\zeta)=\frac{(\zeta-a)^{\beta}}{\beta}$, and $k=1$ in Corollary 2.3, we get the following inequality involving conformable fractional integral operators defined by Jarad $e t$ 
al. [51]:

$$
\begin{aligned}
& { }_{\varrho}^{\beta} \mathfrak{J}^{\varrho}\left(\Upsilon_{1}(\zeta) \Omega_{1}(\zeta)+\Upsilon_{2}(\zeta) \Omega_{2}(\zeta)\right) \chi_{1}(\zeta) \chi_{2}(\zeta) \\
& \quad \geq 4_{\varrho}^{\beta} \mathfrak{J}^{\varrho} \Omega_{1}(\zeta) \Omega_{2}(\zeta) \chi_{1}^{2}(\zeta) \cdot{ }_{\varrho}^{\beta} \mathfrak{J}^{\varrho} \Upsilon_{1}(\zeta) \Upsilon_{2}(\zeta) \chi_{2}^{2}(\zeta)
\end{aligned}
$$

Remark 2.10 Choosing $\beta>0, \psi(\zeta)=\frac{\gamma^{\zeta+5}}{\zeta+5}$, and $k=1$ in Corollary 2.3, we get the results involving conformable fractional integral operators defined by Khan et al. [52], i.e.,

$$
\begin{aligned}
& { }_{\varrho}^{\tau} K_{p^{+}}^{\beta}\left(\Upsilon_{1}(\varsigma) \Omega_{1}(\zeta)+\Upsilon_{2}(\zeta) \Omega_{2}(\zeta)\right) \chi_{1}(\zeta) \chi_{2}(\zeta) \\
& \quad \geq 4_{\varrho}^{\tau} K_{p^{+}}^{\beta} \Omega_{1}(\zeta) \Omega_{2}(\zeta) \chi_{1}^{2}(\zeta) \cdot{ }_{\varrho}^{\tau} K_{p^{+}}^{\beta} \Upsilon_{1}(\zeta) \Upsilon_{2}(\zeta) \chi_{2}^{2}(\zeta) .
\end{aligned}
$$

Lemma 2.11 Let $\chi_{1}, \chi_{2}, \Upsilon_{1}, \Upsilon_{2}, \Omega_{1}$, and $\Omega_{2}$ be positive integrable functions defined on $[0, \infty)$, and $\chi_{1}, \chi_{2}, \Upsilon_{1}, \Upsilon_{2}, \Omega_{1}, \Omega_{2} \in \mathfrak{C}(\mathfrak{h})$ such that (2.1) holds for all $\varsigma \in[0, \gamma]$. Then

$$
\frac{\overline{\Upsilon_{1}(\zeta) \Upsilon_{2}(\zeta)} \overline{\Omega_{1}(\zeta) \Omega_{2}(\zeta)} \overline{\chi_{1}^{2}(\zeta)} \overline{\chi_{2}^{2}(\zeta)}}{\left(\overline{\Upsilon_{1}(\zeta) \chi_{1}(\zeta)} \overline{\Omega_{1}(\zeta) \chi_{2}(\zeta)}+\overline{\Upsilon_{2}(\zeta) \chi_{1}(\zeta)} \overline{\left.\Omega_{2}(\zeta) \chi_{2}(\zeta)\right)^{2}}\right.} \leq \frac{1}{4}
$$

Proof From (2.1), we have the following fact:

$$
\begin{aligned}
& \left(\frac{\Upsilon_{2}(\varsigma)}{\Omega_{1}(\eta)}-\frac{\chi_{1}(\varsigma)}{\chi_{2}(\eta)}\right) \geq 0, \\
& \left(\frac{\chi_{1}(\varsigma)}{\chi_{2}(\eta)}-\frac{\Upsilon_{1}(\varsigma)}{\Omega_{2}(\eta)}\right) \geq 0,
\end{aligned}
$$

which implies that

$$
\left(\frac{\Upsilon_{1}(\varsigma)}{\Omega_{2}(\eta)}+\frac{\Upsilon_{2}(\varsigma)}{\Omega_{1}(\eta)}\right) \frac{\chi_{1}(\varsigma)}{\chi_{2}(\eta)} \geq \frac{\chi_{1}^{2}(\varsigma)}{\chi_{2}^{2}(\eta)}+\frac{\Upsilon_{1}(\varsigma) \Upsilon_{2}(\varsigma)}{\Omega_{1}(\eta) \Omega_{2}(\eta)} .
$$

Multiplying both sides of $(2.26)$ by $\Omega_{1}(\eta) \Omega_{2}(\eta) \chi_{2}^{2}(\eta)$, we obtain

$$
\begin{gathered}
\Upsilon_{1}(\varsigma) \chi_{1}(\varsigma) \Omega_{1}(\eta) \chi_{2}(\eta)+\Upsilon_{2}(\varsigma) \chi_{1}(\varsigma) \Omega_{2}(\eta) \chi_{2}(\eta) \\
\geq \Omega_{1}(\eta) \Omega_{2}(\eta) \chi_{2}^{2}(\varsigma)+\Upsilon_{1}(\eta) \Upsilon_{2}(\eta) \chi_{2}^{2}(\varsigma) .
\end{gathered}
$$

Multiplying (2.27) by $h(\gamma, \zeta) h(\gamma, \eta)$, then integrating with respect to $\zeta$ and $\eta$ over measure space $\Delta$, we obtain

$$
\begin{gathered}
\overline{\Upsilon_{1}(\zeta) \chi_{1}(\zeta)} \overline{\Omega_{1}(\zeta) \chi_{2}(\zeta)}+\overline{\Upsilon_{2}(\zeta) \chi_{1}(\zeta)} \overline{\Omega_{2}(\zeta) \chi_{2}(\zeta)} \\
\geq \overline{\Omega_{1}(\zeta) \Omega_{2}(\zeta)} \overline{\chi_{1}^{2}(\zeta)}+\overline{\Upsilon_{1}(\zeta) \Upsilon_{2}(\zeta)} \overline{\chi_{2}^{2}(\zeta)} .
\end{gathered}
$$

By arithmetic-geometric mean inequality, we obtain

$$
\begin{aligned}
& \overline{\Upsilon_{1}(\zeta) \chi_{1}(\zeta)} \overline{\Omega_{1}(\zeta) \chi_{2}(\zeta)}+\overline{\Upsilon_{2}(\zeta) \chi_{1}(\zeta)} \overline{\Omega_{2}(\zeta) \chi_{2}(\zeta)} \\
& \quad \geq 2 \sqrt{\overline{\Omega_{1}(\zeta) \Omega_{2}(\zeta)} \overline{\chi_{1}^{2}(\zeta)} \overline{\Upsilon_{1}(\zeta) \Upsilon_{2}(\zeta)} \overline{\chi_{2}^{2}(\zeta)}} .
\end{aligned}
$$

This completes the proof. 
Corollary 2.12 Apply Lemma 2.11 with $\Delta=(a, b), d \beta(\varsigma)=d \varsigma$, and $h(\gamma, \varsigma)$ defined by (2.11). Make the following substitutions:

$$
\begin{aligned}
& \overline{\Upsilon_{1}(\zeta) \Upsilon_{2}(\zeta)}=I_{a+; g}^{\rho, k} \Upsilon_{1}(\zeta) \Upsilon_{2}(\zeta), \\
& \overline{\Omega_{1}(\zeta) \Omega_{2}(\zeta)}=I_{a+; g}^{\rho, k} \Omega_{1}(\zeta) \Omega_{2}(\zeta), \\
& \overline{\chi_{1}^{2}(\zeta)}=I_{a+; g}^{\rho, k} \chi_{1}^{2}(\zeta), \\
& \overline{\chi_{2}^{2}(\zeta)}=I_{a+; g}^{\rho, k} \chi_{2}^{2}(\zeta), \\
& \overline{\Upsilon_{1}(\zeta) \chi_{1}(\zeta)}=I_{a+; g}^{\rho, k} \Upsilon_{1}(\zeta) \chi_{1}(\zeta), \\
& \overline{\Omega_{2}(\zeta) \chi_{2}(\zeta)}=I_{a+; g}^{\rho, k} \Omega_{2}(\zeta) \chi_{2}(\zeta), \\
& \overline{\Upsilon_{2}(\zeta) \chi_{1}(\zeta)}=I_{a+; g}^{\rho, k} \Upsilon_{2}(\zeta) \chi_{1}(\zeta),
\end{aligned}
$$

and

$$
\overline{\Omega_{1}(\zeta) \chi_{2}(\zeta)}=I_{a+; g}^{\varrho, k} \Omega_{1}(\zeta) \chi_{2}(\zeta),
$$

we get the following inequality:

$$
\frac{I_{a+; g}^{\rho, k} \Upsilon_{1}(\zeta) \Upsilon_{2}(\zeta) I_{a+g}^{\rho, k} \Omega_{1}(\zeta) \Omega_{2}(\zeta) I_{a+; g}^{\varrho, k} \chi_{1}^{2}(\zeta) I_{a+; g}^{\rho, k} \chi_{2}^{2}(\zeta)}{\left(I_{a+; g}^{\rho, k} \Upsilon_{1}(\zeta) \chi_{1}(\zeta) I_{a+; g}^{\rho, k} \Omega_{1}(\zeta) \chi_{2}(\zeta)+I_{a+; g}^{\rho, k} \Upsilon_{2}(\zeta) \chi_{1}(\zeta) I_{a+; g}^{\rho, k} \Omega_{2}(\zeta) \chi_{2}(\zeta)\right)^{2}} \leq \frac{1}{4}
$$

which is [49, Lemma 3.6].

Remark 2.13 In particular, if we choose $k=1$ in Corollary 2.12, we get [49, Corollary 3.1].

Remark 2.14 Applying Corollary 2.3 with $\psi(\zeta)=\zeta$ leads to the inequality in Corollary 3.4 of [49].

Remark 2.15 Apply Theorem 2.1 with $\Delta=(a, b), d \beta(\varsigma)=d \varsigma$, defined by (2.16). Replacing

$$
\begin{aligned}
& \overline{\Upsilon_{1}(\zeta) \Upsilon_{2}(\zeta)}=J_{a_{+}}^{\varrho} \Upsilon_{1}(\zeta) \Upsilon_{2}(\zeta), \\
& \overline{\Omega_{2}(\zeta) \Omega_{2}(\zeta)}=J_{a_{+}}^{\varrho} \Omega_{2}(\zeta) \Omega_{2}(\zeta), \\
& \overline{\chi_{1}^{2}(\zeta)}=J_{a_{+}}^{\varrho} \chi_{1}^{2}(\zeta), \\
& \overline{\chi_{2}^{2}(\zeta)}=J_{a_{+}}^{\varrho} \chi_{2}^{2}(\zeta), \\
& \overline{\Omega_{2}(\zeta) \chi_{2}(\zeta)}=J_{a_{+}}^{\varrho} \Omega_{2}(\zeta) \chi_{2}(\zeta), \\
& \overline{\Upsilon_{2}(\zeta) \chi_{1}(\zeta)}=J_{a_{+}}^{\varrho} \Upsilon_{2}(\zeta) \chi_{1}(\zeta), \\
& \overline{\Omega_{1}(\zeta) \chi_{2}(\zeta)}=J_{a_{+}}^{\varrho} \Omega_{1}(\zeta) \chi_{2}(\zeta),
\end{aligned}
$$

and

$$
\overline{\Upsilon_{1}(\zeta) \chi_{1}(\zeta)}=J_{a_{+}}^{\varrho} \Upsilon_{1}(\zeta) \chi_{1}(\zeta)
$$


we get the inequality for Hadamard fractional integral, i.e.,

$$
\frac{J_{a_{+}}^{\varrho} \Upsilon_{1}(\zeta) \Upsilon_{2}(\zeta) J_{a_{+}}^{\varrho} \Omega_{1}(\zeta) \Omega_{2}(\zeta) J_{a_{+}}^{\varrho} \chi_{1}^{2}(\zeta) J_{a_{+}}^{\rho} \chi_{2}^{2}(\zeta)}{\left(J_{a_{+}}^{\varrho} \Upsilon_{1}(\zeta) \chi_{1}(\zeta) J_{a_{+}}^{\varrho} \Omega_{1}(\zeta) \chi_{2}(\zeta)+J_{a_{+}}^{\varrho} \Upsilon_{2}(\zeta) \chi_{1}(\zeta) J_{a_{+}}^{\rho} \Omega_{2}(\zeta) \chi_{2}(\zeta)\right)^{2}} \leq \frac{1}{4}
$$

Remark 2.16 Apply Theorem 2.1 with $\Delta=(a, b), d \beta(\varsigma)=d \varsigma, h(\zeta, \varsigma)$ defined by (2.18). Replacing

$$
\begin{aligned}
& \overline{\Upsilon_{1}(\zeta) \Upsilon_{2}(\zeta)}=I_{a_{+} ; \sigma ; \eta}^{\varrho} \Upsilon_{1}(\zeta) \Upsilon_{2}(\zeta), \\
& \overline{\Upsilon_{2}(\zeta) \Upsilon_{2}(\zeta)}=I_{a_{+} ; \sigma ; \eta}^{\rho} \Upsilon_{2}(\zeta) \Upsilon_{2}(\zeta), \\
& \overline{\chi_{2}^{2}(\zeta)}=I_{a_{+} ; \sigma ; \eta}^{\varrho} \chi_{1}^{2}(\zeta), \\
& \overline{\chi_{2}^{2}(\zeta)}=I_{a_{+} ; \sigma ; \eta}^{\varrho} \chi_{2}^{2}(\zeta), \\
& \overline{\Omega_{2}(\zeta) \chi_{2}(\zeta)}=I_{a_{+} ; \sigma ; \eta}^{\varrho} \Omega_{2}(\zeta) \chi_{2}(\zeta), \\
& \overline{\Upsilon_{2}(\zeta) \chi_{1}(\zeta)}=I_{a_{+} ; \sigma ; \eta}^{\varrho} \Upsilon_{2}(\zeta) \chi_{1}(\zeta), \\
& \overline{\Omega_{1}(\zeta) \chi_{2}(\zeta)}=I_{a_{+} ; \sigma ; \eta}^{\varrho} \Omega_{1}(\zeta) \chi_{2}(\zeta),
\end{aligned}
$$

and

$$
\overline{\Upsilon_{1}(\zeta) \chi_{1}(\zeta)}=I_{a_{+} ; \sigma ; \eta}^{\varrho} \Upsilon_{1}(\zeta) \chi_{1}(\zeta)
$$

we get the inequality for Erdélyi-Köber type fractional integral, i.e.,

$$
\frac{I_{a_{+} ; \sigma ; \eta}^{\varrho} \Upsilon_{1}(\zeta) \Upsilon_{2}(\zeta) I_{a_{+} ; \sigma ; \eta}^{\varrho} \Omega_{1}(\zeta) \Omega_{2}(\zeta) I_{a_{+} ; \sigma ; \eta}^{\rho} G^{2}(\zeta) I_{a_{+} ; \sigma ; \eta}^{\varrho} \chi_{2}^{2}(\zeta)}{\left(I_{a_{+} ; \sigma ; \eta}^{\varrho} \Upsilon_{1}(\zeta) \chi_{1}(\zeta) I_{a_{+} ; \sigma ; \eta}^{\varrho} \Omega_{1}(\zeta) \chi_{2}(\zeta)+I_{a_{+} ; \sigma ; \eta}^{\varrho} \Upsilon_{2}(\zeta) \chi_{1}(\zeta) I_{a_{+} ; \sigma ; \eta}^{\rho} \Omega_{2}(\zeta) \chi_{2}(\zeta)\right)^{2}} \leq \frac{1}{4}
$$

Remark 2.17 Choosing $\beta>0, \psi(\zeta)=\frac{\zeta^{\beta}}{\beta}$, and $k=1$ in Corollary 2.12, we get the inequality for the Katugampola fractional integral operator, and the inequality takes the form after replacing

$$
\begin{aligned}
& \overline{\Upsilon_{1}(\zeta) \Upsilon_{2}(\zeta)}={ }^{\rho} I_{a_{+}}^{\rho} \Upsilon_{1}(\zeta) \Upsilon_{2}(\zeta) \\
& \overline{\Upsilon_{2}(\zeta) \Upsilon_{2}(\zeta)}={ }^{\rho} I_{a_{+}}^{\rho} \Upsilon_{2}(\zeta) \Upsilon_{2}(\zeta), \\
& \overline{\chi_{1}^{2}(\zeta)}={ }^{\rho} I_{a_{+}}^{\rho} \chi_{1}^{2}(\zeta) \\
& \overline{\chi_{2}^{2}(\zeta)}={ }^{\rho} I_{a_{+}}^{\rho} \chi_{2}^{2}(\zeta) \\
& \overline{\Omega_{2}(\zeta) \chi_{2}(\zeta)}={ }^{\rho} I_{a_{+}}^{\rho} \Omega_{2}(\zeta) H(\zeta), \\
& \overline{\Upsilon_{2}(\zeta) \chi_{1}(\zeta)}={ }^{\rho} I_{a_{+}}^{\rho} \Upsilon_{2}(\zeta) \chi_{1}(\zeta), \\
& \overline{\Omega_{1}(\zeta) \chi_{2}(\zeta)}={ }^{\rho} I_{a_{+}}^{\rho} \Omega_{1}(\zeta) \chi_{2}(\zeta),
\end{aligned}
$$

and

$$
\overline{\Upsilon_{1}(\zeta) \chi_{1}(\zeta)}={ }^{\rho} I_{a_{+}}^{\varrho} \Upsilon_{1}(\zeta) \chi_{1}(\zeta)
$$


we get

$$
\frac{{ }^{\rho} I_{a_{+}}^{\rho} \Upsilon_{1}(\zeta) \Upsilon_{2}(\zeta)^{\rho} I_{a_{+}}^{\rho} \Omega_{1}(\zeta) \Omega_{2}(\zeta)^{\rho} I_{a_{+}}^{\rho} \chi_{1}^{2}(\zeta)^{\rho} I_{a_{+}}^{\rho} \chi_{2}^{2}(\zeta)}{\left.{ }^{\rho} I_{a_{+}}^{\rho} \Upsilon_{1}(\zeta) \chi_{1}(\zeta)^{\rho} I_{a_{+}}^{\rho} \Omega_{1}(\zeta) \chi_{2}(\zeta)+{ }^{\rho} I_{a_{+}}^{\rho} \Upsilon_{2}(\zeta) \chi_{1}(\zeta)^{\rho} I_{a_{+}}^{\rho} \Omega_{2}(\zeta) \chi_{2}(\zeta)\right)^{2}} \leq \frac{1}{4}
$$

Remark 2.18 Choosing $\beta>0, \psi(\zeta)=\frac{(\zeta-a)^{\beta}}{\beta}$, and $k=1$ in Corollary 2.12, we get the inequality involving conformable fractional integral operators defined by Jarad et al. [51]. Replacing

$$
\begin{aligned}
& \overline{\Upsilon_{1}(\zeta) \Upsilon_{2}(\zeta)}={ }_{\varrho}^{\rho} \mathfrak{J}^{\varrho} \Upsilon_{1}(\zeta) \Upsilon_{2}(\zeta), \\
& \overline{\Upsilon_{2}(\zeta) \Upsilon_{2}(\zeta)}={ }_{\varrho}^{\rho} \mathfrak{J}^{\varrho} \Upsilon_{2}(\zeta) \Upsilon_{2}(\zeta), \\
& \overline{\chi_{1}^{2}(\zeta)}={ }_{\varrho}^{\rho} \mathfrak{J}^{\varrho} \chi_{1}^{2}(\zeta), \\
& \overline{\chi_{2}^{2}(\zeta)}={ }_{\varrho}^{\rho} \mathfrak{J}^{\varrho} \chi_{2}^{2}(\zeta), \\
& \overline{\Omega_{2}(\zeta) \chi_{2}(\zeta)}={ }_{\varrho}^{\rho} \mathfrak{J}^{\varrho} \Omega_{2}(\zeta) \chi_{2}(\zeta), \\
& \overline{\Upsilon_{2}(\zeta) \chi_{1}(\zeta)}={ }_{\varrho}^{\rho} \mathfrak{J}^{\varrho} \Upsilon_{2}(\zeta) \chi_{1}(\zeta), \\
& \overline{\Omega_{1}(\zeta) \chi_{2}(\zeta)}={ }_{\varrho}^{\rho} \mathfrak{J}^{\varrho} \Omega_{1}(\zeta) \chi_{2}(\zeta),
\end{aligned}
$$

and

$$
\overline{\Upsilon_{1}(\zeta) \chi_{1}(\zeta)}={ }_{\varrho}^{\rho} \mathfrak{J}^{\varrho} \Upsilon_{1}(\zeta) \chi_{1}(\zeta)
$$

we get

$$
\frac{{ }_{\varrho}^{\rho} \mathfrak{J}^{\varrho} \Upsilon_{1}(\zeta) \Upsilon_{2}(\zeta)_{\varrho}^{\rho} \mathfrak{J}^{\varrho} \Omega_{1}(\zeta) \Omega_{2}(\zeta)_{\varrho}^{\rho} \mathfrak{J}^{\varrho} \chi_{1}^{2}(\zeta)_{\varrho}^{\rho} \mathfrak{J}^{\varrho} \chi_{2}^{2}(\zeta)}{\left({ }_{\varrho}^{\rho} \mathfrak{J}^{\varrho} \Upsilon_{1}(\zeta) \chi_{1}(\zeta)_{\varrho}^{\rho} \mathfrak{J}^{\varrho} \Omega_{1}(\zeta) \chi_{2}(\zeta)+{ }_{\varrho}^{\rho} \mathfrak{J}^{\varrho} \Upsilon_{2}(\zeta) \chi_{1}(\zeta)_{\varrho}^{\rho} \mathfrak{J}^{\varrho} \Omega_{2}(\zeta) \chi_{2}(\zeta)\right)^{2}} \leq \frac{1}{4}
$$

Remark 2.19 Choosing $\beta>0, \psi(\zeta)=\frac{\zeta^{\xi+\zeta}}{\xi+\varsigma}$, and $k=1$ in Corollary 2.12, we get the inequality for the conformable fractional integral operators defined by Khan et al. [52], and the inequality takes the form by replacing

$$
\begin{aligned}
& \overline{\Upsilon_{1}(\zeta) \Upsilon_{2}(\zeta)}={ }_{\varrho}^{\tau} K_{p^{+}}^{\beta} \Upsilon_{1}(\zeta) \Upsilon_{2}(\zeta), \\
& \overline{\Upsilon_{2}(\zeta) \Upsilon_{2}(\zeta)}={ }_{\varrho}^{\tau} K_{p^{+}}^{\beta} \Upsilon_{2}(\zeta) \Upsilon_{2}(\zeta), \\
& \overline{\chi_{1}^{2}(\zeta)}={ }_{\varrho}^{\tau} K_{p^{+}}^{\beta} G^{2}(\zeta), \\
& \overline{\chi_{2}^{2}(\zeta)}={ }_{\varrho}^{\tau} K_{p^{+}}^{\beta} \chi_{2}^{2}(\zeta), \\
& \overline{\Omega_{2}(\zeta) \chi_{2}(\zeta)}={ }_{\varrho}^{\tau} K_{p^{+}}^{\beta} \Omega_{2}(\zeta) \chi_{2}(\zeta), \\
& \overline{\Upsilon_{2}(\zeta) \chi_{1}(\zeta)}={ }_{\varrho}^{\tau} K_{p^{+}}^{\beta} \Upsilon_{2}(\zeta) \chi_{1}(\zeta), \\
& \overline{\Omega_{1}(\zeta) \chi_{2}(\zeta)}={ }_{\varrho}^{\tau} K_{p^{+}}^{\beta} \Omega_{1}(\zeta) \chi_{2}(\zeta),
\end{aligned}
$$

and

$$
\overline{\Upsilon_{1}(\zeta) \chi_{1}(\zeta)}={ }_{\varrho}^{\tau} K_{p^{+}}^{\beta} \Upsilon_{1}(\zeta) \chi_{1}(\zeta)
$$


we get

$$
\frac{{ }_{\varrho}^{\tau} K_{p^{+}}^{\beta} \Upsilon_{1}(\zeta) \Upsilon_{2}(\zeta)_{\varrho}^{\tau} K_{p^{+}}^{\beta} \Omega_{1}(\zeta) \Omega_{2}(\zeta)_{\varrho}^{\tau} K_{p^{+}}^{\beta} \chi_{1}^{2}(\zeta)_{\varrho}^{\tau} K_{p^{+}}^{\beta} \chi_{2}^{2}(\zeta)}{\left({ }_{\varrho}^{\tau} K_{p^{+}}^{\beta} \Upsilon_{1}(\zeta) \chi_{1}(\zeta){ }_{\varrho}^{\tau} K_{p^{+}}^{\beta} \Omega_{1}(\zeta) \chi_{2}(\zeta)+{ }_{\varrho}^{\tau} K_{p^{+}}^{\beta} \Upsilon_{2}(\zeta) \chi_{1}(\zeta)_{\varrho}^{\tau} K_{p^{+}}^{\beta} \Omega_{2}(\zeta) \chi_{2}(\zeta)\right)^{2}} \leq \frac{1}{4}
$$

Theorem 2.20 Let $\chi_{1}, \chi_{2}, \Upsilon_{1}, \Upsilon_{2}, \Omega_{1}, \Omega_{2} \in \mathfrak{C}(\mathfrak{h})$ be positive integrable functions defined on $[0, \infty)$ such that $(2.1)$ holds for all $\varsigma \in[0, \zeta]$. Then we have

$$
\overline{\left(\frac{\Upsilon_{2} \chi_{1} \chi_{2}}{\Omega_{1}}\right)(\zeta)} \overline{\left(\frac{\Omega_{2} \chi_{1} \chi_{2}}{\Upsilon_{1}}\right)(\zeta)} \geq \overline{\chi_{1}^{2}(\zeta)} \overline{\chi_{2}^{2}(\zeta)}
$$

Proof Clearly from (2.1) we can write

$$
\frac{\Upsilon_{2}(\varsigma)}{\Omega_{1}(\varsigma)} \chi_{1}(\varsigma) \chi_{2}(\varsigma) \geq \chi_{1}^{2}(\varsigma)
$$

which implies that

$$
\int_{\Delta} h(\zeta, \varsigma) \frac{\Upsilon_{2}(\varsigma)}{\Omega_{1}(\varsigma)} \chi_{1}(\varsigma) \chi_{2}(\varsigma) d \beta(\varsigma) \geq \int_{\Delta} h(\zeta, \varsigma) \chi_{1}^{2}(\varsigma) d \beta(\varsigma)
$$

and (2.35) follows.

Corollary 2.21 Apply Theorem 2.20 with $\Delta=(a, b), d \beta(\varsigma)=d \varsigma$, and $h(\zeta, \varsigma)$ defined by (2.11). Replacing

$$
\begin{aligned}
& \overline{\left(\frac{\Upsilon_{2} \chi_{1} \chi_{2}}{\Omega_{1}}\right)(\zeta)}=I_{a+; g}^{\varrho, k}\left(\frac{\Upsilon_{2} \chi_{1} \chi_{2}}{\Omega_{1}}\right)(\zeta), \\
& \overline{\left(\frac{\Omega_{2} \chi_{1} \chi_{2}}{\Upsilon_{1}}\right)(\zeta)}=I_{a+; g}^{\varrho, k}\left(\frac{\Omega_{2} \chi_{1} \chi_{2}}{\Upsilon_{1}}\right)(\zeta), \\
& \overline{\chi_{1}^{2}(\zeta)}=I_{a+; g}^{\varrho, k} \chi_{1}^{2}(\zeta),
\end{aligned}
$$

and

$$
\overline{\chi_{2}^{2}(\zeta)}=I_{a+; g}^{\varrho, k} \chi_{2}^{2}(\zeta)
$$

we get

$$
I_{a+; g}^{\varrho, k}\left(\frac{\Upsilon_{2} \chi_{1} \chi_{2}}{\Omega_{1}}\right)(\zeta) I_{a+; g}^{\varrho, k}\left(\frac{\Omega_{2} \chi_{1} \chi_{2}}{\Upsilon_{1}}\right)(\zeta) \geq I_{a+; g}^{\varrho, k} \chi_{1}^{2}(\zeta) I_{a+; g}^{\varrho, k} \chi_{2}^{2}(\zeta)
$$

which is [49, Theorem 3.11].

Remark 2.22 In particular, if we choose $k=1$ in Corollary 2.21, we get the following inequality:

$$
I_{a+; g}^{\varrho}\left(\frac{\Upsilon_{2} \chi_{1} \chi_{2}}{\Omega_{1}}\right)(\zeta) I_{a+; g}^{\varrho}\left(\frac{\Omega_{2} \chi_{1} \chi_{2}}{\Upsilon_{1}}\right)(\zeta) \geq I_{a+; g}^{\varrho} \chi_{1}^{2}(\zeta) I_{a+; g}^{\varrho} \chi_{2}^{2}(\zeta)
$$

given in [49, Corollary 3.13]. 
Remark 2.23 Applying Corollary 2.21 with $\psi(\zeta)=\zeta$, and corresponding $h(\zeta, \varsigma)$ takes the form given in (2.14) and the inequality takes the form

$$
I_{a+; g}^{\rho, k}\left(\frac{\Upsilon_{2} \chi_{1} \chi_{2}}{\Omega_{1}}\right)(\zeta) I_{a+g}^{\rho, k}\left(\frac{\Omega_{2} \chi_{1} \chi_{2}}{\Upsilon_{1}}\right)(\zeta) \geq I_{a+; g}^{\rho, k} \chi_{1}^{2}(\zeta) I_{a+; g}^{\rho, k} \chi_{2}^{2}(\zeta),
$$

which leads to [49, Corollary 3.14]. Moreover, if we take $k=1$, then (2.38) becomes the inequality given in [36, Lemma 3.1].

Remark 2.24 Apply Theorem 2.20 with $\Delta=(a, b), d \beta(\varsigma)=d \varsigma$, and $h(\zeta, \varsigma)$ defined by (2.16). Replace

$$
\begin{aligned}
& \overline{\left(\frac{\Upsilon_{2} \chi_{1} \chi_{2}}{\Omega_{1}}\right)}=J_{a_{+}}^{\varrho}\left(\frac{\Upsilon_{2} \chi_{1} \chi_{2}}{\Omega_{1}}\right), \\
& \overline{\left(\frac{\Omega_{2} \chi_{1} \chi_{2}}{\Upsilon_{1}}\right)}=J_{a_{+}}^{\varrho}\left(\frac{\Omega_{2} \chi_{1} \chi_{2}}{\Upsilon_{1}}\right), \\
& \overline{\chi_{1}^{2}(\zeta)}=J_{a_{+}}^{\varrho} \chi_{1}^{2}(\zeta),
\end{aligned}
$$

and

$$
\overline{\chi_{2}^{2}(\zeta)}=J_{a_{+}}^{\varrho} \chi_{2}^{2}(\zeta)
$$

to get the inequality for the Hadamard type fractional integrals, i.e.,

$$
J_{a_{+}}^{\varrho}\left(\frac{\Upsilon_{2} \chi_{1} \chi_{2}}{\Omega_{1}}\right)(\zeta) J_{a_{+}}^{\varrho}\left(\frac{\Omega_{2} \chi_{1} \chi_{2}}{\Upsilon_{1}}\right)(\zeta) \geq J_{a_{+}}^{\varrho} \chi_{1}^{2}(\zeta) J_{a_{+}}^{\varrho} \chi_{2}^{2}(\zeta)
$$

Remark 2.25 Apply Theorem 2.21 with $\Delta=(a, b), d \beta(\varsigma)=d \varsigma$, and $h(\zeta, \varsigma)$ defined by (2.18). Replacing

$$
\begin{aligned}
& \overline{\left(\frac{\Upsilon_{2} \chi_{1} \chi_{2}}{\Omega_{1}}\right)(\zeta)}=I_{a_{+} ; \sigma ; \eta}^{\varrho}\left(\frac{\Upsilon_{2} \chi_{1} \chi_{2}}{\Omega_{1}}\right)(\zeta), \\
& \overline{\left(\frac{\Omega_{2} \chi_{1} \chi_{2}}{\Upsilon_{1}}\right)(\zeta)}=I_{a_{+} ; \sigma ; \eta}^{\varrho}\left(\frac{\Omega_{2} \chi_{1} \chi_{2}}{\Upsilon_{1}}\right)(\zeta), \\
& \overline{\chi_{1}^{2}(\zeta)=I_{a_{+} ; \sigma ; \eta}^{\varrho} \chi_{1}^{2}(\zeta),}
\end{aligned}
$$

and

$$
\overline{\chi_{2}^{2}(\zeta)}=I_{a_{+} ; \sigma ; \eta}^{\varrho} \chi_{2}^{2}(\zeta)
$$

we get the results involving Erdélyi-Köber fractional integral, i.e.,

$$
I_{a_{+} ; \sigma ; \eta}^{\varrho}\left(\frac{\Upsilon_{2} \chi_{1} \chi_{2}}{\Omega_{1}}\right)(\zeta) I_{a_{+} ; \sigma ; \eta}^{\varrho}\left(\frac{\Omega_{2} \chi_{1} \chi_{2}}{\Upsilon_{1}}\right)(\zeta) \geq I_{a_{+} ; \sigma ; \eta}^{\varrho} \chi_{1}^{2}(\zeta) I_{a_{+} ; \sigma ; \eta}^{\varrho} \chi_{2}^{2}(\zeta) .
$$


Remark 2.26 Choosing $\beta>0, \psi(\zeta)=\frac{\zeta^{\beta}}{\beta}$, and $k=1$ in Corollary 2.21, we get the inequality for the Katugampola fractional integral operators [53], and the inequality takes the form

$$
{ }^{\rho} I_{a_{+}}^{\varrho}\left(\frac{\Upsilon_{2} \chi_{1} \chi_{2}}{\Omega_{1}}\right)(\zeta)^{\rho} I_{a_{+}}^{\varrho}\left(\frac{\Omega_{2} \chi_{1} \chi_{2}}{\Upsilon_{1}}\right)(\zeta) \geq{ }^{\rho} I_{a_{+}}^{\varrho} \chi_{1}^{2}(\zeta)^{\rho} I_{a_{+}}^{\varrho} \chi_{2}^{2}(\zeta)
$$

Remark 2.27 Choosing $\beta>0, \psi(\zeta)=\frac{(\zeta-a)^{\beta}}{\beta}$, and $k=1$ in Corollary 2.21, we get the inequality for the conformable fractional integral operators defined by Jarad et al. [51], and the inequality takes the form:

$$
{ }_{\varrho}^{\rho} \mathfrak{J}^{\varrho}\left(\frac{\Upsilon_{2} \chi_{1} \chi_{2}}{\Omega_{1}}\right)(\zeta)_{\varrho}^{\rho} \mathfrak{J}^{\varrho}\left(\frac{\Omega_{2} \chi_{1} \chi_{2}}{\Upsilon_{1}}\right)(\zeta) \geq{ }_{\varrho}^{\rho} \mathfrak{J}^{\varrho} \chi_{1}^{2}(\zeta)_{\varrho}^{\rho} \mathfrak{J}^{\varrho} \chi_{2}^{2}(\zeta)
$$

Remark 2.28 Choosing $\beta>0, \psi(\zeta)=\frac{(\zeta)^{\xi+\zeta}}{\xi+\varsigma}$, and $k=1$ in Corollary 2.21, we get the inequality for the conformable fractional integral operators defined by Khan et al. [52], and the inequality takes the form

$$
{ }_{\varrho}^{\tau} K_{p^{+}}^{\beta}\left(\frac{\Upsilon_{2} \chi_{1} \chi_{2}}{\Omega_{1}}\right)(\zeta)_{\varrho}^{\tau} K_{p^{+}}^{\beta}\left(\frac{\Omega_{2} \chi_{1} \chi_{2}}{\Upsilon_{1}}\right)(\zeta) \geq{ }_{\varrho}^{\tau} K_{p^{+}}^{\beta} \chi_{1}^{2}(\zeta)_{\varrho}^{\tau} K_{p^{+}}^{\beta} \chi_{2}^{2}(\zeta)
$$

\section{3 Čebyšev type inequalities}

In this section, we shall present several Čebyšev type inequalities involving general kernels and application in fractional integrals.

Theorem 3.1 Let $\chi_{1}$ and $\chi_{2}$ be two integrable and synchronous functions on $[0, \infty)$ and $\chi_{1}, \chi_{2} \in \mathfrak{C}(\mathfrak{h})$. Then one has

$$
\overline{\left(\chi_{1} \chi_{2}\right)(\zeta)} \geq \frac{1}{\Upsilon(\zeta)} \overline{\chi_{1}(\zeta)} \overline{\chi_{2}(\zeta)}
$$

where $\Upsilon$ is defined by (1.3).

Proof By using the synchronism property for the functions $\chi_{1}$ and $\chi_{2}$ on the interval $[0, \infty)$, we get

$$
\chi_{1}(\zeta) \chi_{2}(\zeta)+\chi_{1}(\varsigma) \chi_{2}(\varsigma) \geq \chi_{1}(\zeta) \chi_{2}(\varsigma)+\chi_{1}(\varsigma) \chi_{2}(\zeta)
$$

Multiplying by $h(\zeta, \varsigma)$ and integrating with respect to $\varsigma$ over measure space $\Delta$ leads to

$$
\begin{aligned}
& \int_{\Delta} h(\zeta, \varsigma) \chi_{1}(\varsigma) \chi_{2}(\varsigma) d \beta(\varsigma)+\int_{\Delta} h(\zeta, \varsigma) \chi_{1}(\xi) \chi_{2}(\xi) d \beta(\varsigma) \\
& \quad \geq \int_{\Delta} h(\zeta, \varsigma) \chi_{1}(\zeta) \chi_{2}(\xi) d \beta(\varsigma)+\int_{\Delta} h(\zeta, \varsigma) \chi_{1}(\xi) \chi_{2}(\varsigma) d \beta(\varsigma) .
\end{aligned}
$$

Therefore, we get that

$$
\overline{\left(\chi_{1} \chi_{2}\right)(\zeta)}+\chi_{1}(\xi) \chi_{2}(\xi) \Upsilon(\zeta) \geq \chi_{2}(\xi) \overline{\chi_{1}(\zeta)}+\chi_{1}(\xi) \overline{\chi_{2}(\zeta)}
$$


Multiplying by $h(\zeta, \xi)$ and integrating with respect to $\xi$ over $\Delta$ leads to

$$
\Upsilon(\zeta) \overline{\left(\chi_{1} \chi_{2}\right)(\zeta)}+\overline{\chi_{1}(\zeta) \chi_{2}(\zeta)} \Upsilon(\zeta) \geq \overline{\chi_{2}(\zeta) \chi_{1}(\zeta)}+\overline{\chi_{2}(\zeta)} \overline{\chi_{1}(\zeta)}
$$

That implies (3.1).

Corollary 3.2 Applying Theorem 3.1 with $\Delta=(a, b), d \beta(\varsigma)=d \varsigma$, and $h(\zeta, \varsigma)$ defined by (2.11), after some calculations, we get

$$
\Upsilon(\zeta)=\frac{1}{\Gamma_{k}(\varrho+k)}(\psi(\zeta)-\psi(a))^{\frac{\varrho}{k}}
$$

Replacing $\overline{\left(\chi_{1} \chi_{2}\right)(\zeta)}=I_{a+; \psi}^{\rho, k}\left(\chi_{1} \chi_{2}\right)(\zeta), \overline{\chi_{1}(\zeta)}=I_{a+; \psi}^{e, k} \chi_{1}(\zeta)$, and $\overline{\chi_{2}(\zeta)}=I_{a+; \psi}^{\rho, k} \chi_{2}(\zeta)$, we get

$$
I_{a+; \psi}^{\varrho, k}\left(\chi_{1} \chi_{2}\right)(\zeta) \geq \frac{1}{\Upsilon(\zeta)} I_{a+; \psi}^{\varrho, k} \chi_{1}(\zeta) \cdot I_{a+; \psi}^{\varrho, k} \chi_{2}(\zeta)
$$

which is [49, Theorem 4.1].

Remark 3.3 In particular, if we choose $k=1$ in Corollary 3.2, we get [49, Corollary 4.2].

Remark 3.4 In particular, if we choose $\psi(\zeta)=\zeta$ in Corollary 3.2, we get [49, Corollary 4.3].

Remark 3.5 In particular, if we choose $\psi(\zeta)=\zeta, k=1$ in Corollary 3.2, we get [49, Corollary 4.4].

Remark 3.6 In particular, if we choose $\psi(\zeta)=\log (\zeta), k=1$ in Corollary 3.2, it leads to the inequality for Katugampola fractional integral operator [53].

Remark 3.7 If we choose $\beta>0, \psi(\zeta)=\frac{(\zeta-a)^{\beta}}{\beta}$, and $k=1$ in Corollary 3.2, we get the inequality for the conformable fractional integral operator [51].

Remark 3.8 Choosing $\beta>0, \psi(\zeta)=\frac{\zeta^{\xi+5}}{\xi+\varsigma}$, and $k=1$ in Corollary 3.2, we get the inequality for the conformable fractional integral operator [52].

Theorem 3.9 Let $\chi_{j},(1 \leq j \leq \gamma)$ be integrable and synchronous functions on $[0, \infty)$ and $\chi_{j} \in \mathfrak{C}(\mathfrak{h})$. Then

$$
\overline{\prod_{j=1}^{\gamma} \chi_{j}}(\zeta) \leq\left(\frac{1}{\Upsilon(\zeta)}\right) \prod_{j=1}^{\gamma} \overline{\chi_{j}}
$$

Proof Proof follows from the mathematical induction.

Remark 3.10 Like previous remarks we can give the applications for fractional integrals discussed in the paper. But we omit the details here. 


\section{Concluding remarks}

In the ongoing years numerous analysts have given the generalization of integral operators and constructed fruitful inequalities. For us it is always interesting and motivating to give the generalization of all previous results. Inspired by the above-mentioned results, we introduced certain rich inequalities successfully, which generalized all the previous results. We developed a class of functions representing the integral transform with general kernel. We proved a wide range of Pólya-Szegö and Čebyšev type inequalities involving general kernel over $\sigma$-finite measure. We extracted the known results from our general results.

\section{Acknowledgements}

All authors are thankful to the careful referee and the editor for their suggestions which improved the final version of our paper. The author T. Abdeljawad would like to thank Prince Sultan University for funding this work through research group Nonlinear Analysis Methods in Applied Mathematics (NAMAM) group number RG-DES-2017-01-17.

\section{Funding}

Not applicable.

Availability of data and materials

Not applicable.

\section{Competing interests}

The authors declare that they have no competing interests.

\section{Authors' contributions}

The authors have worked equally when writing this paper. All authors read and approved the final manuscript.

\section{Author details}

${ }^{1}$ Department of Mathematics, Riphah International University, Faisalabad Campus, Satyana Road, Faisalabad, Pakistan. ${ }^{2}$ Department of Mathematics, University of Sargodha, Sargodha, Pakistan. ${ }^{3}$ Department of Mathematics and General Sciences, Prince Sultan University, Riyadh 12345, Saudi Arabia. ${ }^{4}$ Department of Medical Research, China Medical University, Taichung 40402, Taiwan. ${ }^{5}$ Department of Computer Science and Information Engineering, Asia University, Taichung, Taiwan. ${ }^{6}$ Department of Mathematics, College of Arts and Sciences, Prince Sattam bin Abdulaziz University, Wadi Aldawaser, 11991, Saudi Arabia. ${ }^{7}$ Department of Mathematics and Statistics, Hazara University, Mansehra 21120 ,

Pakistan. ${ }^{8}$ Department of Mathematics, University of Peshawar, Peshawar, Pakistan.

\section{Publisher's Note}

Springer Nature remains neutral with regard to jurisdictional claims in published maps and institutional affiliations.

Received: 9 August 2020 Accepted: 23 November 2020 Published online: 30 November 2020

\section{References}

1. Adjabi, Y., Jarad, F., Baleanu, D., Abdeljawad, T.: On Cauchy problems with Caputo Hadamard fractional derivatives, Math. Methods Appl. Sci. 40(11), 661-681 (2016)

2. Chu, Y.-M., Adil Khan, M., Ali, T., Dragomir, S.S.: Inequalities for a-fractional differentiable functions. J. Inequal. Appl. 2017, Article ID 93 (2017)

3. Adil Khan, M., Iqbal, A., Suleman, M., Chu, Y.-M.: Hermite-Hadamard type inequalities for fractional integrals via Green's function. J. Inequal. Appl. 2018, Article ID 161 (2018)

4. Adil Khan, M., Khurshid, Y., Du, T.-S., Chu, Y.-M.: Generalization of Hermite-Hadamard type inequalities via conformable fractional integrals. J. Funct. Spaces 2018, Article ID 5357463 (2018)

5. Khurshid, Y., Adil Khan, M., Chu, Y.-M., Khan, Z.A.: Hermite-Hadamard-Fejér inequalities for conformable fractional integrals via preinvex functions. J. Funct. Spaces 2019, Article ID 3146210 (2019)

6. Tan, W., Jiang, F.-L., Huang, C.-X., Zhou, L.: Synchronization for a class of fractional-order hyperchaotic system and its application. J. Appl. Math. 2012, Article ID 974639 (2012)

7. Wu, J., Liu, Y.-C.: Uniqueness results and convergence of successive approximations for fractional differential equations. Hacet. J. Math. Stat. 42(2), 149-158 (2013)

8. Huang, C.-X., Liu, L.-Z.: Sharp function inequalities and boundedness for Toeplitz type operator related to general fractional singular integral operator. Publ. Inst. Math. 92(106), 165-176 (2012)

9. Zhou, X.-S., Huang, C.-X., Hu, H.-J., Liu, L.: Inequality estimates for the boundedness of multilinear singular and fractional integral operators. J. Inequal. Appl. 2013, Article ID 303 (2013)

10. Liu, F.-W., Feng, L.-B., Anh, V., Li, J.: Unstructured-mesh Galerkin finite element method for the two-dimensional multi-term time-space fractional Bloch-Torrey equations on irregular convex domains. Comput. Math. Appl. 78(5), $1637-1650(2019)$

11. Jiang, Y.-J., $\mathrm{Xu}, \mathrm{X}$.-J.: A monotone finite volume method for time fractional Fokker-Planck equations. Sci. China Math. 62(4), $783-794(2019)$

12. Zhou, S.-H., Jiang, Y.-J.: Finite volume methods for N-dimensional time fractional Fokker-Planck equations. Bull. Malays. Math. Sci. Soc. 42(6), 3167-3186 (2019) 
13. Kilbas, A.A., Srivastava, H.M., Trujillo, J.J.: Theory and Applications of Fractional Differential Equations. Elsevier, Amsterdam (2006)

14. Cai, Z.-W., Huang, J.-H., Huang, L.-H.: Periodic orbit analysis for the delayed Filippov system. Proc. Am. Math. Soc. 146(11), 4667-4682 (2018)

15. Chen, T., Huang, L.-H., Yu, P., Huang, W.-T.: Bifurcation of limit cycles at infinity in piecewise polynomial systems. Nonlinear Anal., Real World Appl. 41, 82-106 (2018)

16. Duan, L., Fang, X.-W., Huang, C.-X.: Global exponential convergence in a delayed almost periodic Nicholson's blowflies model with discontinuous harvesting. Math. Methods Appl. Sci. 41(5), 1954-1965 (2018)

17. Tan, Y.-X., Huang, C.-X., Sun, B., Wang, T.: Dynamics of a class of delayed reaction-diffusion systems with Neumann boundary condition. J. Math. Anal. Appl. 458(2), 1115-1130 (2018)

18. Wang, J.-F., Chen, X.-Y., Huang, L.-H.: The number and stability of limit cycles for planar piecewise linear systems of node-saddle type. J. Math. Anal. Appl. 469(1), 405-427 (2019)

19. Wang, J.-F., Huang, C.-X., Huang, L.-H.: Discontinuity-induced limit cycles in a general planar piecewise linear system of saddle-focus type. Nonlinear Anal. Hybrid Syst. 33, 162-178 (2019)

20. Huang, C.-X., Zhang, H., Huang, L.-H.: Almost periodicity analysis for a delayed Nicholson's blowflies model with nonlinear density-dependent mortality term. Commun. Pure Appl. Anal. 18(6), 3337-3349 (2019)

21. Hu, H.-J., Zou, X.-F.: Existence of an extinction wave in the Fisher equation with a shifting habitat. Proc. Am. Math. Soc. 145(11), 4763-4771 (2017)

22. Tang, W.-S., Sun, Y.-J.: Construction of Runge-Kutta type methods for solving ordinary differential equations. Appl. Math. Comput. 234, 179-191 (2014)

23. Xie, D.-X., Li, J.: A new analysis of electrostatic free energy minimization and Poisson-Boltzmann equation for protein in ionic solvent. Nonlinear Anal., Real World Appl. 21, 185-196 (2015)

24. Dai, Z.-F., Chen, X.-H., Wen, F.-H.: A modified Perry's conjugate gradient method-based derivative-free method for solving large-scale nonlinear monotone equations. Appl. Math. Comput. 270, 378-386 (2015)

25. Feng, L.-B., Zhuang, P., Liu, F., Turner, I., Anh, V., Li, J.: A fast second-order accurate method for a two-sided space-fractional diffusion equation with variable coefficients. Comput. Math. Appl. 73(6), 1155-1171 (2017)

26. Li, J., Liu, F., Fang, L., Turner, l.: A novel finite volume method for the Riesz space distributed-order diffusion equation. Comput. Math. Appl. 74(4), 772-783 (2017)

27. Wang, W.-S.: Fully-geometric mesh one-leg methods for the generalized pantograph equation: approximating Lyapunov functional and asymptotic contractivity. Appl. Numer. Math. 117, 50-68 (2017)

28. Liu, Z.-Y., Wu, N.-C., Qin, X.-R., Zhang, Y.-L.: Trigonometric transform splitting methods for real symmetric Toeplitz systems. Comput. Math. Appl. 75(8), 2782-2794 (2018)

29. Li, J., Ying, J.-Y., Xie, D.-X.: On the analysis and application of an ion size-modified Poisson-Boltzmann equation. Nonlinear Anal., Real World Appl. 47, 188-203 (2019)

30. Agarwal, P.: Some inequalities involving Hadamard-type k-fractional integral operators. Math. Methods Appl. Sci. 40(11), 3882-3891 (2017)

31. Agarwal, P.: Certain properties of the generalized Gauss hypergeometric functions. Appl. Math. Inf. Sci. 8(5), 2315-2320 (2014)

32. Agarwal, P., Choi, J.: Certain fractional integral inequalities associated with pathway fractional integral operators. Bull. Korean Math. Soc. 53(1), 181-193 (2016)

33. Agarwal, P., Jain, S., Mansour, T.: Further extended Caputo fractional derivative operator and its applications. Russ. J. Math. Phys. 24(4), 415-425 (2017)

34. Agarwal, P., Jleli, M., Tomar, M.: Certain Hermite-Hadamard type inequalities via generalized k-fractional integrals. J. Inequal. Appl. 2017, Article ID 55 (2017)

35. Grüss, G.: Uber das Maximum des absoluten Betrages $\frac{1}{b-a} \int_{a}^{b} f(x) g(x) d x-\frac{1}{(b-a)^{2}} \int_{a}^{b} f(x) d x \int_{a}^{b} g(x) d x$. Math. Z. 39(1), 215-226 (1935)

36. Ntouyas, S., Agarwal, P., Tariboon, J.: On Pólya-Szegö and Chebyshev types inequalities involving the Riemann-Liouville fractional integral operators. J. Math. Inequal. 10(2), 491-504 (2016)

37. Ozdemir, M.E., Set, E., Akdemir, A.O., Sarikaya, M.Z.: Some new Chebyshev type inequalities for functions whose derivatives belongs to Lp spaces. Afr. Math. 26(7-8), 1609-1619 (2015)

38. Abdeljawad, T., Mohammed, P.O., Kashuri, A.: New modified conformable fractional integral inequalities of Hermite-Hadamard type with applications. J. Funct. Spaces 2020, Article ID 4352357 (2020) https://doi.org/10.1155/2020/4352357

39. Mohammed, P.O.: Hermite-Hadamard inequalities for Riemann-Liouville fractional integrals of a convex function with respect to a monotone function. Math. Methods Appl. Sci. 1-11 (2019). https://doi.org/10.1002/mma.5784

40. Set, E., Akdemir, A.O., Mumcu, I.: Hadamard's inequality and its extensions for conformable fractional integrals of any order $a>0$. Creative Math. Inform. 27(2), 197-206 (2018)

41. Agarwal, P.: Fractional integration of the product of two multivariables $\mathrm{H}$-function and a general class of polynomials. In: Advances in Applied Mathematics and Approximation Theory. Springer Proc. Math. Stat., vol. 41, pp. 359-374. Springer, New York (2013)

42. Chebyshev, P.L.: Sur les expressions approximatives des integrales definies par les autres prises entre les mêmes limites. Proc. Math. Soc. Charkov 2, 93-98 (1882)

43. Pólya, G., Szegö, G.: Aufgaben und Lehrstze aus der Analysis i. Springer, New York (1964)

44. Dragomir, S.S., Diamond, N.T.: Integral inequalities of Grüss type via Pólya-Szegö and Shisha-Mond results. East Asian Math. J. 19(1), 27-39 (2003)

45. Samko, S.G., Kilbas, A.A., Marichev, O.I.: Fractional Integrals and Derivatives: Theory and Applications. Gordon \& Breach, Yverdon (1993)

46. Diaz, R., Pariguan, E: On hypergeometric functions and Pochhammer k-symbol. Divulg. Mat. 15, 179-192 (2007)

47. Mubeen, S., Habibullah, G.M.: k-Fractional integrals and application. Int. J. Contemp. Math. Sci. 7(2), 89-94 (2012)

48. Kilbas, A.A., Srivastava, H.M., Trujillo, J.J.: Theory and Applications of Fractional Differential Equations. North-Holland Mathematics Studies, vol. 204. Elsevier, New York (2006) 
49. Rashid, S., Jarad, F., Kalsoom, H., Chu, Y.: On Pólya-Szegö and Cebyšev type inequalities via generalized k-fractional integrals. Adv. Differ. Equ. 2020, 125 (2020)

50. Samko, S.G., Kilbas, A.A., Marichev, O.I. Fractional Integrals and Derivatives: Theory and Applications. Gordon \& Breach, New York (1993)

51. Jarad, F., Ugurlu, E., Abdeljawad, T., Baleanu, D.: On a new class of fractional operators. Adv. Differ. Equ. 2017, Article ID 247 (2017)

52. Khan, T.U., Adil Khan, M.: Generalized conformable fractional operators. J. Comput. Appl. Math. 346, 378-389 (2019)

53. Katugampola, U.N.: New fractional integral unifying six existing fractional integrals. arXiv:1612.08596 [math.CA]

Submit your manuscript to a SpringerOpen ${ }^{\circ}$ journal and benefit from:

- Convenient online submission

- Rigorous peer review

- Open access: articles freely available online

- High visibility within the field

- Retaining the copyright to your article

Submit your next manuscript at $\gg$ springeropen.com 